\title{
Liver Ultrasonographic Elastography
}

National Cancer Institute

\section{Source}

National Cancer Institute. Liver Ultrasonographic Elastography. NCI Thesaurus. Code C138323.

A noninvasive test that is used to stage fibrosis in the liver. A 50-MHz wave is passed into the liver from a small transducer on the end of an ultrasound probe which measures the velocity of the shear wave (in meters per second) as this wave passes through the liver. The shear wave velocity can then be converted into liver stiffness. This technique is an alternative to liver biopsy. 